\title{
Closed Reduction of Displaced Mandibular Fracture in a Child - A Case Study
}

\author{
Dr. Thinakar Babu .A \\ Senior Lecturer, The TN Dr MGR Medical University, Chennai, Tamilnadu, India
}

\begin{abstract}
Mandibular fractures in the pediatric population are uncommon. Principles involved in the treatment of children are the same, but techniques get modified by anatomical, physiological and psychological factors. The conservative treatment is in most cases advocated, as this also has minimum effort to the surgeon and can be done in conscious child along with risk benefit and cost benefit concerning childs socioeconomic profile. This article we present the management of mandibular fracture in a $2 \frac{1}{2}$ years child who was managed with manual reduction then direct wiring.
\end{abstract}

Keywords: paediatric mandibular fracture, dental wiring, figure of eight wiring

\section{Introduction}

Pediatric fractures are rare when compared with fractures in the adult population. The reported incidence of pediatric injuries accounts for $4-6 \%$ of the total. Below the age of 5 years, the incidence of pediatric facial fractures is even lower, ranging from 0.6 to $1.2 \%$. Injuries may be due to falls, assault, sports related injuries and automobile accidents. In the management of these fractures, the goal is to restore the underlying bony architecture to its pre-injury position in a stable fashion withminimal aesthetic and functional impairement. Closed reduction technique with maxillomandibular fixation in very young children can pose several concerns, including co-operation, compliance and adequate nutritional intake. Open reduction with rigid internal fixation (ORIF) of unstable mandibular fractures using miniplates and screws are thought to have a negative effect on skeletal growth and unerupted teeth and involve two stage surgery because of the need for plate removal after complete healing. An occlusal splint and circummandibular wire is another treatment for mandibular fractures in children. ${ }^{1}$

Depending on the type of fracture and the stage of skeletal development the treatment modalities range from conservative, non - invasive through closed reduction and immobilization methods to open reduction with internal fixation. Distruption of the periosteal envelope of the mandibular body may have an unpredictable effect on growth. Thus, if reduction is required, closed reduction is favored. $^{2}$

\section{Case Report}

A $21 / 2$ years old male child came to casualty with the history of alleged assault by his drunken father holding both legs of child and hit the face to the wall of the room.Multidicplinary team allowed us to intervene as the simultaneous head hit is of no concern as per the neurologist advice.

The patient medical history was noncontributory and he was not under any medication. Patient was conscious,alert and awake. Clinical and radiographic evaluation showed a through and through laceration on chin region about $2 \times 1 \mathrm{~cm}$ in size. Condyle was palpable bilaterally. Step deformity present in the lower border of the mandible. Occlusal derangement and Sublingual ecchymosis was present.

CT shows displaced fracture line passing between 71 and 72 and track downwards and backwards to the base of the mandible \& bilateral fracture of head of the condyle.Regarding golden hour of pediatric dental fracture should be intervened within atleast four hours Primary closure of the laceration was done with 3-0vicryl. Displaced left parasymphysis fracture was snaped back into the place by manual closed reduction. Immobization of reduced fracture segment was done with direct wiring (figure of eight) under LA and left for three weeks. The antibiotic treatment consisted of Amoxicillin oral suspension $(25 \mathrm{mg} / \mathrm{kg}$ three times a day).Acetoaminophen suspension (three times a day $250 \mathrm{mg}$ ).Patient was advised soft diet then recalled after three weeks, the healing was satisfactory. patient was on follow up. Two years following the incident the patients mouth opening and occlusion was normal, no facial asymmetry was noted. OPG and occlusal radiographs showed no deformity in the mandible.

\section{Discussion}

Fractures of the pediatric facial skeleton have special characteristics, and specific knowledge is required for their diagnosis, management and follow-up. To understand the difference between the pediatric and adult facial fracture patterns, a familiarity with the process of facial development is essential. Facial growth, paranasal sinus development, dentition and bone structure all affect the pattern of facial structures in children. ${ }^{3}$

A green stick fracture is a fracture in which one cortex of the bone is broken and the other cortex is bend. Green stick fracture commonly occur in children due to thin cortices, inherent elasticity of bone, thick surrounding layer of adipose tissue and the relatively larger amount of medullary bone held by a strong periosteal support results in a high incidence of greenstick fractures in children. ${ }^{4}$

Reparative process in children is rapid due to the increased metabolic rate and high osteogenic potential of periosteum. This result in early union, usually occur within 3 weeks, and delay in treatment for any reason can cause more serious problems than in adults.

Volume 6 Issue 1, January 2017 


\section{International Journal of Science and Research (IJSR) \\ ISSN (Online): 2319-7064}

Index Copernicus Value (2015): 78.96 | Impact Factor (2015): 6.391

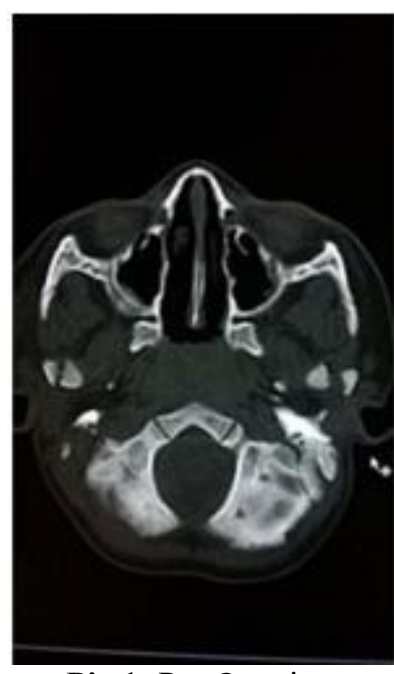

Pic 1: Pre Opening

In 2-4 years child sufficient well formed, sound deciduous teeth are present, so interdental wiring can be used. If there are gaps in the dentition an arch bar may be required or cap splint can be used. But cap splint should be avoided, because cementing of splints and their subsequent removal and cleaning of the teeth necessitates considerable cooperation between operator and the child. The closed reduction and immobilization can be achieved by acrylic splints, circumferential wiring.

ORIF in children is preffered only if other means of reduction and fixation are not attainable. Problems of ORIF in pediatric patients are presence of developing tooth germs. Though the inferior border of mandible can be plated, interference with growth, allergic reactions to metal can occur due to presence of miniplates. So currently ORIF with resorbable plates and screws are increasingly being used in children. ${ }^{5}$

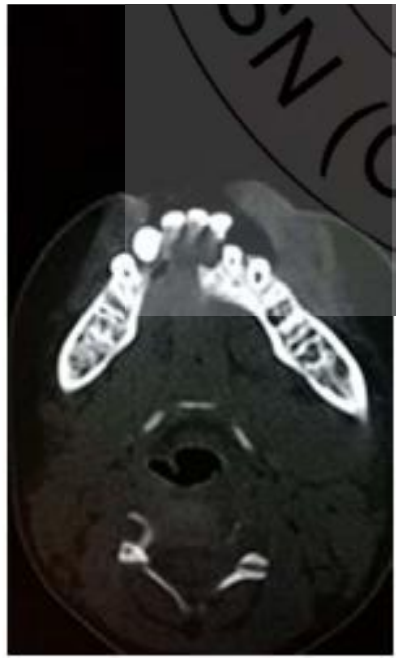

Pic 2 - CT Mandible

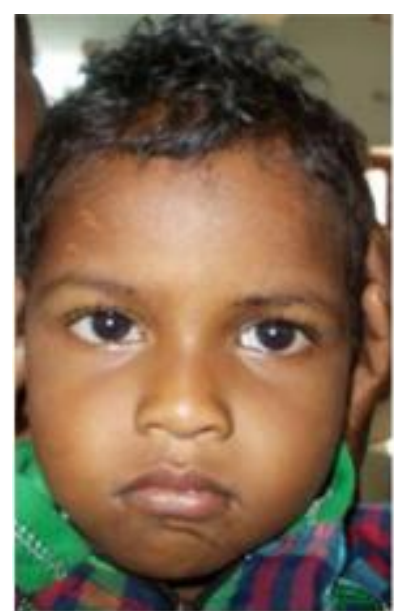

Pic 5: Post Operation

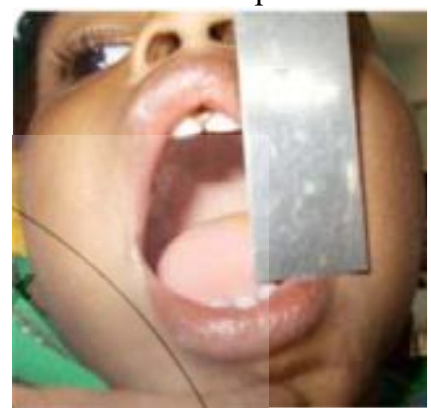

Pic 6: Mouth Opening

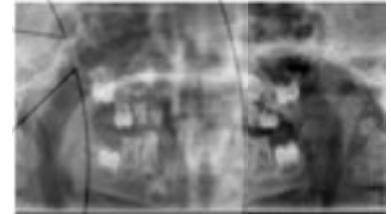

Pic 7: OP 2 yr Post Operation

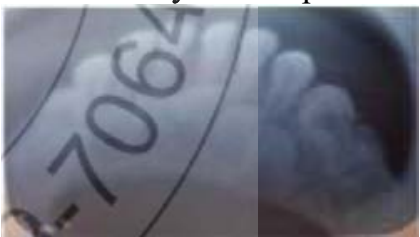

Pic 8: Occlasal view

\section{Conclusion}

While the basic principles for mandibular fracture treatment are the same as for the adult, certain anatomical features of the pediatric mandible warrant special attention. In cases of mandibular fractures of a young child, disruption of periosteal envelope may have an unpredictable effects on growth. Thus effective, simplest and less invasive method is the best method for children. Also regarding multidiciplinary approach of pediatric facial fractures if there is simultaneous head hit along with maxillofacial fracture, treatment much be given preferably to area of interest which is more important at that time with prior permission from the specialists concerned.

\section{References}

[1] Raymond J. Fonseca,Robert v.walker. Oral and maxillofacial trauma, $2^{\text {nd }}$ edition W.B.saunders, Philadelphia, p.p1001-1010,1997. 


\section{International Journal of Science and Research (IJSR) \\ ISSN (Online): 2319-7064}

Index Copernicus Value (2015): 78.96 | Impact Factor (2015): 6.391

[2] Rowe and Williams.maxillofacial injuries, $2^{\text {nd }}$ ed. 12. Reddy BK, Barttett SP. Pediatric facial Fractures. p.p387-400,1996.

[3] Alcala-galianoA, arribas-garcia IJ, Martin - perez Ma peadiatric facial fractures: children are not just small adults. 2008;28: 441-461.

[4] Crean ST, sirarajasingam v. conservative management of mandibular fractures in the early dentition phase. A case report and review of the literature .Int $\mathrm{J}$ pediatr dent2000;10:229-33.

[5] Aizenbud D , harzan -malina $\mathrm{k}$. the management of mandibular body fractures in young children - dental traumatol 2009;25:569-70

[6] kaban LD diagnosis and treatment of fractures of facial bones in children 1943-1993.oral and maxillofacial surgery 1993;51:722-9

[7] LasterZ,muscura EA.Pediatric mandibular fractures.Introduction of a novel therapeutic modality. J trauma 2008;64:225-9.

[8] Rowe NL. Fracture of the jaws in children. J Oral Surg 1969;27:497-507.

[9] Schiller E, Bratu E. Traumatic injuries to the primary teeth and their impact on permanent teeth. TimmisoaraMedial Journal 2003.p.2.

[10] Gaven O. Fractures of the maxillofacial region in children. J CraniomaxillofacSurg 1992;20:244-7.

[11] Schweinfurth JM, Koltai PJ. Pediatric mandibular fractures. Facial plastsurg 1998;14:31-44.

[12] Pediatric plastic surgery.Ed.Stamford.p.p.463-86, 1998. 